\title{
Feasibility and efficacy of modified fixed citrate concentration protocol using only commercial preparations in critically ill patients: a prospective cohort study with a historical control group
}

\author{
Yan Shi ${ }^{*}$, Han-Yu Qin, Jin-Min Peng, Xiao-Yun Hu and Bin Du
}

\begin{abstract}
Background: The cumbersome program and the shortage of commercial solution hindered the regular application of regional citrate anticoagulation (RCA). It is urgent to simplify the protocol using only commercial preparations. The aim of this study was to explore the feasibility and efficacy of the modified protocol for continuous venovenous hemofiltration $(\mathrm{CWH})$ in unselected critically ill patients.

Methods: A prospective cohort study was conducted in 66 patients who received a new protocol combining fixed citrate concentration with modified algorithm for supplements (i.e., fixed protocol), and compared the efficacy, safety and convenience for this group to a historical control group with a traditional protocol $(n=64)$, where citrate was titrated according to the circuit ionized calcium concentration (i.e., titrated protocol). The convenience was defined as the demand for monitoring test and dose adjustment of any supplement.

Results: The filter lifespan was $63.2 \pm 16.1 \mathrm{~h}$ in the fixed group and $51.9 \pm 17.7 \mathrm{~h}$ in the titrated group, respectively. Kaplan-Meier survival analysis demonstrated longer circuit lifetime for fixed group (log-rank, $p=0.026$ ). The incidence of circuit clotting was lower in the fixed protocol (15.2\% vs. $29.7 \%$ in the titrated protocol, $p=0.047)$. Moreover, compared with the titrated group, patients with fixed protocol had less demand for monitoring test and dose adjustment of any supplement (the number of times per person per day) (3.3 [IQR 2.3-4.5] vs. 5.7 [IQR 3.3-6.9], $p=0.001$ and 1.9 [IQR 0.5-2.7] vs. 6.3 [IQR 4.2-7.9], $p<0.001$; respectively). No new onset bleeding complications occurred in all patients. The overall incidence of suspected citrate accumulation was $4.6 \%$ and there was no difference between the two groups $(p=0.969)$, yet a lower rate of metabolic alkalosis was found in the fixed group (3.0\% vs. $14.1 \%, p=0.024)$.

* Correspondence: pumchshi@sina.com

Department of medical ICU, Peking Union Medical College Hospital, Peking Union Medical College and Chinese Academy of Medical Sciences, 1 Shuai

Fu Yuan, Dongcheng District, Beijing 100730, China

(c) The Author(s). 2021 Open Access This article is licensed under a Creative Commons Attribution 4.0 International License, which permits use, sharing, adaptation, distribution and reproduction in any medium or format, as long as you give appropriate credit to the original author(s) and the source, provide a link to the Creative Commons licence, and indicate if changes were made. The images or other third party material in this article are included in the article's Creative Commons licence, unless indicated otherwise in a credit line to the material. If material is not included in the article's Creative Commons licence and your intended use is not permitted by statutory regulation or exceeds the permitted use, you will need to obtain permission directly from the copyright holder. To view a copy of this licence, visit http://creativecommons.org/licenses/by/4.0/ The Creative Commons Public Domain Dedication waiver (http://creativecommons.org/publicdomain/zero/1.0/) applies to the data made available in this article, unless otherwise stated in a credit line to the data. 
(Continued from previous page)

Conclusions: Our modified fixed citrate concentration protocol is feasible, safe and effective to enhance the circuit lifespan and the convenience of implementation while maintaining a similar safety when compared to the traditional protocol. Using only commercial preparations may be helpful for widespread application of RCA.

Trial registration: Clinicaltrials.gov. NCT02663960. Registered 26 January 2016.

Keywords: Regional citrate anticoagulation (RCA), Continuous veno-venous hemofiltration (CWH), Commercial preparations, Protocol, Filter lifespan, Convenience, Critically ill patients

\section{Background}

Continuous renal replacement therapy (CRRT) is an important tool in the care of critically ill patients, and adequate anticoagulation is needed to prevent circuit clotting. Kidney Disease Improved Global Outcomes (KDIGO) guideline suggests regional citrate anticoagulation (RCA) as the first-line method in patients without contraindication for citrate [1]. However, in clinical practice, the use of RCA is still limited because of concerns related to the risk of metabolic complications and the complexity of the proposed protocols [2-5]. In particular, a shortage of commercial solution and high costs also hinder its regular application in developing countries. For example, in China, clinicians prefer heparin to RCA for continuous veno-venous hemofiltration $(\mathrm{CVVH})$, and the major factors limiting the use of citrate are high cost (32\%), complex program (21\%), limited source of commercial solution (18\%) and concerns of complications (12\%) [6]. So, it is urgent to simplify the protocol using only commercial preparations.

Nowadays, the most frequently used method is a separate citrate infusion combined with pre or post-dilution replacement fluid (RF) [7-12]. This scheme needs to adjust at least three solutions according to the mechanism of citrate anticoagulation and its metabolic [2-5]: (1) the amount of citrate needs to be titrated according to the circuit ionized calcium (c-iCa) levels; (2) a systemic calcium infusion replaces the calcium loss in the effluent and (3) citrate as a buffer substrate may require adjustment of bicarbonate supplement. Also, strict laboratory monitoring is needs to prevent serious metabolic complications. Therefore, the traditional scheme increases complexity, labor intensive and iatrogenic errors.

More recently, RCA protocol has been simplified by using citrate-buffered RF in predilution mode, which is isotonic and acts as an anticoagulant and buffer [13-20]. However, since CVVH dose was coupled to citrate dose, the inflexibility in flow rates changes and insufficient dialysis dose were its main disadvantages [3, 5, 21]. In addition, the assumption of using citrate-buffered solution instead of additional buffer infusion was limited by variable alkali load provided by citrate in the different CRRT mode, which was different between amount of citrate infusion and loss into the effluent (e.g, CVVH, continuous veno venous hemodialysis [CVVHD] or continuous veno venous hemodiafiltration [CVVHDF]) $[2,5$, $22,23]$, in clinical practice, it was not sufficient to compensate for acidosis, so an additional infusion of bicarbonate was required [16-18]. Also, since anticoagulation was coupled with metabolic control, the buffer cannot be titrated separately and limited the ability to fine tune the control of metabolic disturbances $[5,21$, 22]. Besides, the citrate-buffered solution has to be custom-made, increasing cost and labour. Although a dilute citrate solution has recently become available in Europe known as prismocitrate 10/2, anticoagulant citrate dextrose $(\mathrm{ACD})$ solution and bicarbonate buffered fluid are the common used in most countries. Moreover, most medical centers in China have to use calciumcontaining RF due to an absence of commercially available calcium-free solution $[6,9,10]$.

In order to widely employ RCA, we devised a new protocol using fixed circuit citrate concentration combined with modified algorithm for supplements (i.e., fixed protocol) for CVVH in unselected adult critically ill patients, and compared the efficacy, safety and convenience for this group to a historical control group with a traditional titrated protocol.

\section{Methods}

\section{Study design and selection of patients and controls}

This prospective study was conducted at a 15-bed medical intensive care unit (ICU) of the Peking Union Medical College Hospital, Beijing, China. After 2 months of staff training, from August 2015, our institution began to employ a new protocol as part of the routine method for CVVH.

We prospectively recruited patients older than 18 years of age who required RCA-CVVH. Patients were excluded if they:1) required systemic heparin anticoagulation (SHA), because RCA will no longer be adopted according to our usual practice. On the contrary, the subcutaneous injection of low molecular weight heparin (LMWH), as a routine prophylactic or therapeutic method for deep venous thrombosis in patients with low risk of bleeding, does not affect the choice of anticoagulant method for $\mathrm{CVVH}$ and the recruitment of patients; 2) had incomplete data; 3) had CVVH running time less 
than $6 \mathrm{~h}$. Historical control group of patients who met the same criteria have been screened backward since August 2015.

This study was approved by the institution's Research Ethics Board (No. ZS-950), and written informed consent was obtained from all prospective study participants or their relatives.

\section{RCA-CVVH protocols}

Before and after implementation of a new protocol, predilution CVVH was performed using same commercially anticoagulant citrate dextrose solution -A (ACDA) $\left(\mathrm{Na}^{+} 224\right.$, citrate 113 , bicarbonate 203 all in $\mathrm{mmol} / \mathrm{L}$, Nigale Biotechnology Co. Ltd., Sichuan, China) and calcium- containing alkali-free $\mathrm{RF}\left(\mathrm{Na}^{+} 113, \mathrm{Cl}^{-} 118, \mathrm{Mg}\right.$ ${ }^{2+} 0.797, \mathrm{Ca}^{2+} 1.60$ all in $\mathrm{mmol} / \mathrm{L}$; Qingshan Likang, Pharmaceutical Co. Ltd., Chengdu, China). The same standards were adopted, such as CRRT devices (Aquarius or Diapact), catheter type (a double-lumen 12-F catheter, Arrow International Inc., USA), vascular access (placed in the internal jugular or femoral vein) and haemofilter (DIACAP Acute L, $2.0 \mathrm{~m}^{2}$, B. Braun Melsungen AG, Germany). The circuit was run for $72 \mathrm{~h}$ unless there was filter clotting, transmembranous pressure exceeded 300 $\mathrm{mmHg}$, transportation was required, or the predefined clinical target was reached. The separate ACD-A was administered in the "arterial" line of the extracorporeal circuit. The flow rate of RF was flexibly adjusted to achieve a prescribed dialysis dose of at least $25 \mathrm{ml} / \mathrm{kg} / \mathrm{h}$. Calcium gluconate (10\%) and sodium bicarbonate solutions (5\%) were infused through the return line of the circuit.

For the titrated protocol, ACD-A was initiated with $2.5 \%$ of blood flow, corresponding to $3 \mathrm{mmol} / \mathrm{L}$ of extracorporeal blood (eg, blood flow is typically $7200 \mathrm{ml} / \mathrm{h}$ [120 ml/min] and ACD-A is run at $180 \mathrm{ml} / \mathrm{h}$ ) $[8,9,20]$. Then, the citrate flow was adjusted to maintain the c$\mathrm{iCa}$ of 0.2 to $0.4 \mathrm{mmol} / \mathrm{L}$, as described in previous studies $[8,9,19,20]$. The initial calcium substitution flow was set at $6.1 \%$ of ACD-A flow and titrated to maintain the systemic ionized calcium (s-iCa) of 1.0 to $1.2 \mathrm{mmol} / \mathrm{L}$ (see Additional file 1). The initial infusion rate of sodium bicarbonate was set at $5 \%$ of RF flow (equivalent to $\mathrm{HCO}^{-}$concentration of $30 \mathrm{mmol} / \mathrm{L}$ in the RF), and then was adjusted to keep blood $\mathrm{pH}$ within the normal range.

The fixed protocol has been modified in three aspects: First, considering predilution calcium- containing solution increased calcium load in vitro (e.g., the calcium load increased by $20-30 \%$ when the RF flow rate was usually set at $2-2.5 \mathrm{l} / \mathrm{h}$ ) $[5,9]$, we determined a target citrate concentration of $4 \mathrm{mmol} / \mathrm{L}$ instead of the lowest concentration reported of $3 \mathrm{mmol} / \mathrm{L}$ [8, 9, 20, 23]. ACDA infusion dose corresponding to blood flow was shown in Additional file 2. This constant concentration was maintained during CRRT unless citrate accumulation (CA) was suspected, which were resolved by reducing either blood flow or circuit citrate concentration until discontinuation. Second, calcium substitution flow was determined through a roughly estimate the amount of calcium loss at $0.8 \mathrm{mmol}$ per liter total effluent flow based on the preliminary experiment results involving 10 patients (see Additional file 3). For patients with hypocalcemia at start $\mathrm{CVVH}$, a bolus dose of calcium gluconate was delivered $(0.3 \mathrm{ml} / \mathrm{kg})$. Third, bicarbonate substitution flow was set at $3.5 \%$ of RF flow (corresponding to $\mathrm{HCO}^{-}$of $20 \mathrm{mmol} / \mathrm{L}$ in the $\mathrm{RF}$ ), which was determined by using a mathematical model to roughly estimate the alkali load provided by citrate (i.e., citrate metabolic load $(\mathrm{mmol} / \mathrm{h})=[$ citrate solution concentration $(\mathrm{mmol} / \mathrm{L}) \times$ citrate flow rate $(\mathrm{l} / \mathrm{h})]-$ [effluent rate $(\mathrm{l} / \mathrm{h}) \mathrm{x}$ estimated citrate blood concentration $(\mathrm{mmol} / \mathrm{L}) \mathrm{x}$ sieving coefficients of citrate]) [20]. If necessary, the infusion rate of calcium or bicarbonate solution should also be adjusted to hold s-iCa and $\mathrm{pH}$ within the normal range.

The same monitoring algorithm was used in the two groups. The first measurements of $\mathrm{c}-\mathrm{iCa}, \mathrm{s}-\mathrm{iCa}, \mathrm{pH}$ and $\mathrm{HCO}^{-}$levels were done $1 \mathrm{~h}$ after initiation of $\mathrm{CVVH}$ and every 4 to $6 \mathrm{~h}$ during the first $24 \mathrm{~h}$. Then these measurements were done according to clinical needs. Moreover, c-iCa level was measured $1 \mathrm{~h}$ after any change in blood flow, citrate, RF or calcium flow rate. The liver and kidney functions, haemoglobin, platelet counts, total serum calcium, prothrombin time and activated partial thromboplastin time were measured at least daily.

\section{Data collection}

All data were collected through the hospital information system and the prescriptions for $\mathrm{CVVH}$, and included patient's age, sex, comorbidity, severity score using the acute physiology and chronic health evaluation (APAC HE) II and the sequential organ failure assessment (SOFA) score, application of LMWH, physiological support, reasons for start and stopping $\mathrm{CVVH}$, filter lifetime, CVVH settings (e.g., blood flow, ACD-A flow, RF flow and effluent flow), frequency of dose adjustment for any supplement, laboratory variables and monitoring requirements during $\mathrm{CVVH}$ and ICU mortality at 28 days.

\section{Study endpoints and definition}

The primary endpoint was filter lifespan of the first circuit. The reasons for stopping CVVH were divided elective discontinuation (e.g., replacement per protocol at $72 \mathrm{~h}$, clinical target achievement, CA, death or discharge) or spontaneous circuit failure (circuit clotting, transmembrane pressure $>300 \mathrm{mmHg}$, catheter malfunction) $[12,14]$. The secondary endpoint was the convenience, which was defined as the demand for monitoring 
tests and dose adjustment of any supplement per person per day. We also compared the incidence of complications, including severe metabolic disorders (defined as metabolic alkalosis with $\mathrm{pH}>7.5$, metabolic acidosis with $\mathrm{pH}<7.25$, hypocalcemia with $\mathrm{s}-\mathrm{iCa}<0.7 \mathrm{mmol} / \mathrm{L}$, hypercalcemia with total serum calcium $\geq 2.75 \mathrm{mmol} / \mathrm{L}$, hypernatremia with $\mathrm{Na} \geq 150 \mathrm{mmol} / \mathrm{L}$ ), bleeding disorders (defined as the decline of hemoglobin $10 \mathrm{~g} / \mathrm{L}$ within $12 \mathrm{~h}$ ) and suspected CA (identified as a total calcium/ionic calcium ratio $\geq 2.5$ ), as described in the literature $[17,19,20]$.

\section{Sample size and statistics}

We hypothesized a difference of $10 \mathrm{~h}$ in mean circuit survival for different RCA protocol, with an estimated mean circuit survival of $40 \mathrm{~h}$ and pooled standard deviation of $20 \mathrm{~h}$, which was reported by some randomized clinical trial and our experience [12, 17, 19]. To obtain $80 \%$ power with a two-sided $\alpha$ level $<0.05$, the sample size was targeted at 63 patients per group and final sample size at 70 patients per group is needed to correct for an expected $10 \%$ drop out.

Continuous variables were presented as means and standard deviation (SD) for normally distributed data, and medians and interquartile range (IQR) for all other data, whereas categorical variables were presented as number (percentage). Continuous variables were compared with the use of the Student's t test or MannWhitney test, while categorical variables were compared using chi-square test or Fisher's exact test. The KaplanMeier survival curve was used to depict the cumulative survival, and a log-rank test was used to assess the difference. All comparisons were unpaired, and all tests of significance were two-tailed. A $p$ value $<0.05$ was considered statistically significant. The statistical analyses were performed with SPSS statistics software (version 20.0; SPSS Inc., Chicago, IL).

\section{Result}

\section{Clinical characteristics and CVVH implementation}

We consecutively screened 140 patients forward (until May 2016) and backward (until September 2014). Four patients (one of requiring SHA and 3 of running time $<6 \mathrm{~h}$ ) in a prospective session and 6 patients $(2$ of insufficient data and 4 of running time $<6 \mathrm{~h}$ ) in a retrospective phase were excluded, and 130 patients were included in the final analysis (64 in the titrated group and 66 in the fixed group).

The mean age was $63.1 \pm 15.9$ years old and the median body weight was $70.7 \mathrm{~kg}$. Twenty-five patients had a history of chronic kidney dysfunction (stage 3 or greater according to KDIGO) and 8 had dialysis-dependent; Two patients had liver dysfunction with Child-Pugh B or C. The most common reason for CVVH was due to sepsisinduced acute kidney injury, accounting for $83.8 \%$. Fortythree patients (33.1\%) received subcutaneous LMWH treatment. The APACHE II score was $22.3 \pm 4.7$. One hundred and 18 patients $(90.8 \%)$ required mechanical ventilation, 115 patients $(88.5 \%)$ received vasopressors. ICU mortality rate at 28 days was $32.3 \%(n=42)$. Demographic, clinical characteristics and laboratory examination at baseline were well-matched across groups (Table 1).

There was no significant difference between the two groups in terms of blood flow, RF and total effluent flow rate (Table 2). As expected by the protocol, a significant decrease in the initial infusion rate $(\mathrm{ml} / \mathrm{h})$ of calcium and bicarbonate was observed in the fixed group than that in the titrated group (9.5 vs.12.1, $p=0.038$ and 70 vs.105, $p=0.015$; respectively). Also, the initial ACD-A flow $(\mathrm{ml} / \mathrm{h})$ of the fixed group was slightly higher than that of the titrated group ( $261 \pm 13$ vs $191 \pm 11, p=$ $0.037)$, but was similar at the end of CVVH $(259 \pm 13$ vs. $242 \pm 15, p=0.378$ ). Serum creatinine, blood urea nitrogen and potassium levels were significantly decreased with $\mathrm{CVVH}$ therapy and there were similar between the two groups (Table 2).

\section{Filter lifetime and c-iCa monitoring parameters}

The total running time of the fixed group and the titrated group was $3266 \mathrm{~h}$ and $4336 \mathrm{~h}$, respectively. The circuit lifetime was $63.2 \pm 16.1 \mathrm{~h}$ with fixed protocol and $51.9 \pm 17.7 \mathrm{~h}$ with titrated protocol $(p=0.014)$. Ninetyone percent, 85 and $68 \%$ of the hemofilters with fixed protocol still operated at 24,48 , and $72 \mathrm{~h}$, respectively, while 81,69 and $53 \%$ in the titrated group, respectively. The Kaplan-Meier survival analysis demonstrated longer circuit lifetime for fixed group (log-rank, $p=0.026$ ) (Fig. 1). The rate of circuit failure due to clotting in the fixed group was lower than that in the titrated group (15.2\% vs $29.7 \%, p=0.047)$, but there was no difference in the termination rate due to other reasons (Table 3). Even for the clotted filters, the circuit lifespans were significantly prolonged by the fixed protocol, when compared to the titrated scheme $(48.7 \pm 15.4 \mathrm{~h}$ vs $36.5 \pm 18.4$ h, $p=0.044$ ).

During CRRT, the c-iCa levels in 525 and 714 blood samples of the fixed group and the titrated group were measured respectively. The first measured concentration of $\mathrm{c}-\mathrm{iCa}$ was $0.33 \pm 0.05 \mathrm{mmol} / \mathrm{L}$ in the fixed group, $98.5 \%$ of which was within the target range of $0.2-0.4$ $\mathrm{mmol} / \mathrm{L}$, while that was $0.38 \pm 0.09 \mathrm{mmol} / \mathrm{L}$ and $90.6 \%$ in the titrated group, respectively $(p<0.001$ and $p=0.031$, respectively). Of all measured $\mathrm{c}-\mathrm{iCa}, 1.5 \%$ exceeded 0.4 $\mathrm{mmol} / \mathrm{L}, 10.6 \%$ had $\mathrm{c}-\mathrm{iCa}$ values at 0.35 to $0.4 \mathrm{mmol} / \mathrm{L}$, $84.8 \%$ was at 0.2 to $0.34 \mathrm{mmol} / \mathrm{L}$ and $3.1 \%$ was less than $0.2 \mathrm{mmol} / \mathrm{L}$ in the fixed group, while $7.7,46.9,42.5$ and $2.9 \%$ were found in the titrated group, respectively $(p<0.001)$. Also, the medium $\mathrm{c}-\mathrm{iCa}$ levels $(\mathrm{mmol} / \mathrm{L})$ in the fixed protocol were lower than that in the titrated protocol (0.31 [IQR 0.25-0.36] vs 0.37 [IQR 0.29-0.39], $p=0.002)$. 
Table 1 The patients' characteristics and laboratory parameters at CWH start between the two groups

\begin{tabular}{|c|c|c|c|}
\hline Variables & Fixed group $(n=66)$ & Titrated group $(n=64)$ & $P$ value \\
\hline Age, years, mean (SD) & $62.7(18.4)$ & $64.1(17.6)$ & 0.379 \\
\hline Female gender, n (\%) & $24(36.4)$ & $22(34.4)$ & 0.813 \\
\hline Body weight, kg, median (IQR) & $69.2(61.5-74.3)$ & $70.5(63.5-74.5)$ & 0.852 \\
\hline \multicolumn{4}{|l|}{ Comorbidities, n (\%) } \\
\hline Chronic kidney disease $^{a}$ & $13(19.7)$ & $12(18.8)$ & 0.891 \\
\hline Dialysis-dependent & $6(9.1)$ & $2(3.1)$ & 0.157 \\
\hline Diabetes mellitus & $14(21.2)$ & $17(26.6)$ & 0.474 \\
\hline Hypertension & $19(28.8)$ & $16(25.0)$ & 0.626 \\
\hline Liver dysfunction ${ }^{b}$ & $1(1.5)$ & $1(1.6)$ & 0.983 \\
\hline Cardiac insufficiency $^{c}$ & $3(4.5)$ & $3(4.7)$ & 0.969 \\
\hline \multicolumn{4}{|l|}{ Severity of illness } \\
\hline APACHE II score, mean (SD) & $23.1(6.8)$ & $22.7(6.4)$ & 0.541 \\
\hline SOFA score, mean (SD) & $8.0(2.7)$ & $8.3(2.3)$ & 0.672 \\
\hline Vasopressors, n (\%) & $58(87.9)$ & $57(89.1)$ & 0.833 \\
\hline Mechanical ventilation, n (\%) & $60(90.9)$ & $58(90.6)$ & 0.955 \\
\hline \multicolumn{4}{|l|}{ Indication for CWH, n (\%) } \\
\hline Sepsis-induced AKI & $54(81.8)$ & $55(85.9)$ & 0.523 \\
\hline Fluid overload & $5(7.6)$ & $5(7.8)$ & 0.960 \\
\hline Tumorlysis syndrome & $4(6.1)$ & $2(3.1)$ & 0.425 \\
\hline Others & $3(4.5)$ & $2(3.1)$ & 0.674 \\
\hline Application of LMWH, n (\%) & 19(28.8) & $24(37.5)$ & 0.352 \\
\hline \multicolumn{4}{|l|}{ Laboratory parameters } \\
\hline Hemoglobin, g/L, mean (SD) & $89.1(19.2)$ & $85.5(19.7)$ & 0.573 \\
\hline Platelet count, $10^{9} / \mathrm{L}$, median (IQR) & $74.1(50.7-97.5)$ & $81.5(60.8-102.2)$ & 0.153 \\
\hline APTT, S, mean (SD) & $42.6(8.7)$ & $43.2(8.1)$ & 0.754 \\
\hline PT, S, mean (SD) & $14.0(2.7)$ & $14.6(2.3)$ & 0.805 \\
\hline Serum albumin, g/L, mean (SD) & $23.3(2.4)$ & $24.7(3.5)$ & 0.672 \\
\hline Bilirubin, mmol/l, mean (SD) & $29.4(7.5)$ & $27.0(10.8)$ & 0.718 \\
\hline $\mathrm{ALT}, \mathrm{U} / \mathrm{L}$, mean (SD) & $68(28.8)$ & $56(25.5)$ & 0.182 \\
\hline Creatinine, $\mu \mathrm{mol} / \mathrm{l}$, median (IQR) & $229(169-559)$ & $240(143-497)$ & 0.756 \\
\hline Urea, mmol/l, median (IQR) & $33.9(15.6)$ & $30.8(14.7)$ & 0.347 \\
\hline Lactate, mmol/l, median (IQR) & $4.8(2.7-6.8)$ & $4.5(3.1-6.3)$ & 0.498 \\
\hline ICU death at 28 days, $\mathrm{n}(\%)$ & $20(30.3)$ & $22(34.4)$ & 0.239 \\
\hline
\end{tabular}

Abbreviations: AKI acute renal injury, ALT Alanine Aminotransferease, APACHE, acute physiology and chronic health evaluation, APTT activated partial thromboplastin time, $C V V H$ continuous veno-venous hemofiltration, ICU Intensive Care Unit; IQR interquartile range, $L M W H$ low molecular weight heparin, $P T$ prothrombin time, $R C A$ regional citrate anticoagulation, SD standard deviation, SOFA Sequential Organ Failure Assessment

${ }^{a}$ Chronic kidney disease defined as stage 3-5 according to Kidney Disease Improving Global outcome in 2012;

b liver dysfunction defined as Child-pugh B-C stage

c Chronic cardiac insufficiency defined of stage III-IV according to New York Heart Association

\section{Convenience of implementation}

During CVVH, more frequent adjustment (the number of times per person, per day) of any infusion pump was found in the titrated group (6.3 [IQR 4.2-7.9] vs. 1.9 [IQR 0.5-2.7], p<0.001). Among them, the adjustment frequency of calcium and bicarbonate infusion pump in the titrated group versus the fixed group were 2.5 (IQR 2.0-4.1) vs. 0.5 (IQR $0.3-0.9$ ) and 3.5 (IQR 2.5-4.8) vs.
1.5 (IQR $0.8-2.7$ ), respectively (all $p<0.05$ ). In particular, $87.5 \%$ of patients with titrated protocol needed to adjust the dosages of ACD-A infusion pump, with a change of 28.5 (IQR $12.8-43.5$ ) $\mathrm{ml} / \mathrm{h}$, but only two patients suspected of CA were adjusted in the fixed group. In addition, 703 and 1096 blood samples were taken for monitoring tests during CRRT in the fixed group and the titrated group, respectively, and patients with fixed 
Table 2 CWH setting, acid-base and metabolic control at CWH start and end between groups

\begin{tabular}{|c|c|c|c|c|}
\hline \multirow[t]{2}{*}{ Variables } & \multicolumn{2}{|l|}{ Fixed group } & \multicolumn{2}{|l|}{ Titrated group } \\
\hline & Start & End $^{a}$ & Start & End $^{a}$ \\
\hline \multicolumn{5}{|l|}{ CWH settings, mean (SD) } \\
\hline Blood flow (ml/min) & $122(7)$ & $122(6)$ & $123(10)$ & $122(8)$ \\
\hline Citrate flow $(\mathrm{ml} / \mathrm{h})$ & $261(13)^{*}$ & $259(13)$ & $191(11)$ & $242(15)$ \\
\hline Replacement flow (ml/h) & $2148(102)$ & $2218(175)$ & $2292(122)$ & $2198(103)$ \\
\hline Total effluent flow (ml/h) & $2743(270)$ & 2612(347) & $2698(470)$ & 2745 (229) \\
\hline Sodium bicarbonate (ml/h) & $70(10)^{*}$ & $60(25)$ & $105(25)$ & $63(23)^{* *}$ \\
\hline Calcium gluconate (ml/h) & $9.5(1.2)^{*}$ & $8.3(2.7)$ & $12.1(1.2)$ & $8.1(3.4)$ \\
\hline \multicolumn{5}{|l|}{ Acid-base status } \\
\hline $\mathrm{pH}$, mean (SD) & $7.29(0.1)^{* *}$ & $7.39(0.1)$ & $7.26(0.1)^{* *}$ & $7.42(0.2)$ \\
\hline Bicarbonate, mean (SD) & $19.3(4.1)^{* *}$ & $25.5(3.5)^{*}$ & $18.7(3.5)^{* *}$ & $27.9(4.2)$ \\
\hline Base Excess, median (IQR) & $-6.0(-6.5,-5.4)^{* *}$ & $1.7(-0.5,2.9)^{*}$ & $-5.7(-6.7,-5.3)^{* *}$ & $4.1(-0.7,4.7)$ \\
\hline \multicolumn{5}{|l|}{ Metabolic control } \\
\hline Serum creatinine, $\mu \mathrm{mol} / \mathrm{L}$ & $229(169-559)^{* *}$ & $136(85-178)$ & $240(143-497)^{* *}$ & $148(93-185)$ \\
\hline Blood urea nitrogen, $\mathrm{mmol} / \mathrm{L}$ & $33.9(15.6)^{* *}$ & $22.1(9.8)$ & $30.8(14.7)^{* *}$ & $20.3(6.7)$ \\
\hline Sodium, mmol/L & $138.7(3.5)$ & $140.1(5.7)$ & $138.5(5.8)$ & $141.0(5.2)$ \\
\hline Potassium, mmol/L & $5.4(1.8)^{* *}$ & $3.9(1.3)$ & $5.3(1.5)^{* *}$ & $3.9(1.3)$ \\
\hline Total serum calcium, mmol/L & $2.12(0.3)$ & $2.10(0.2)$ & $2.13(0.2)$ & $2.12(0.3)$ \\
\hline lonized calcium, mmol/L & $1.07(0.15)$ & $1.05(0.14)$ & $1.08(0.13)$ & $1.06(0.08)$ \\
\hline
\end{tabular}

Abbreviations: $C V V H$ continuous veno-venous hemofiltration, $I Q R$ interquartile range, $S D$ standard deviation

${ }^{*}: p<0.05$ for fixed vs titrated group; ${ }^{* *}: p<0.05$ for CVVH start vs end in the each group

${ }^{a}$ : The data at the end of $\mathrm{CVVH}$ comes from the average of the day

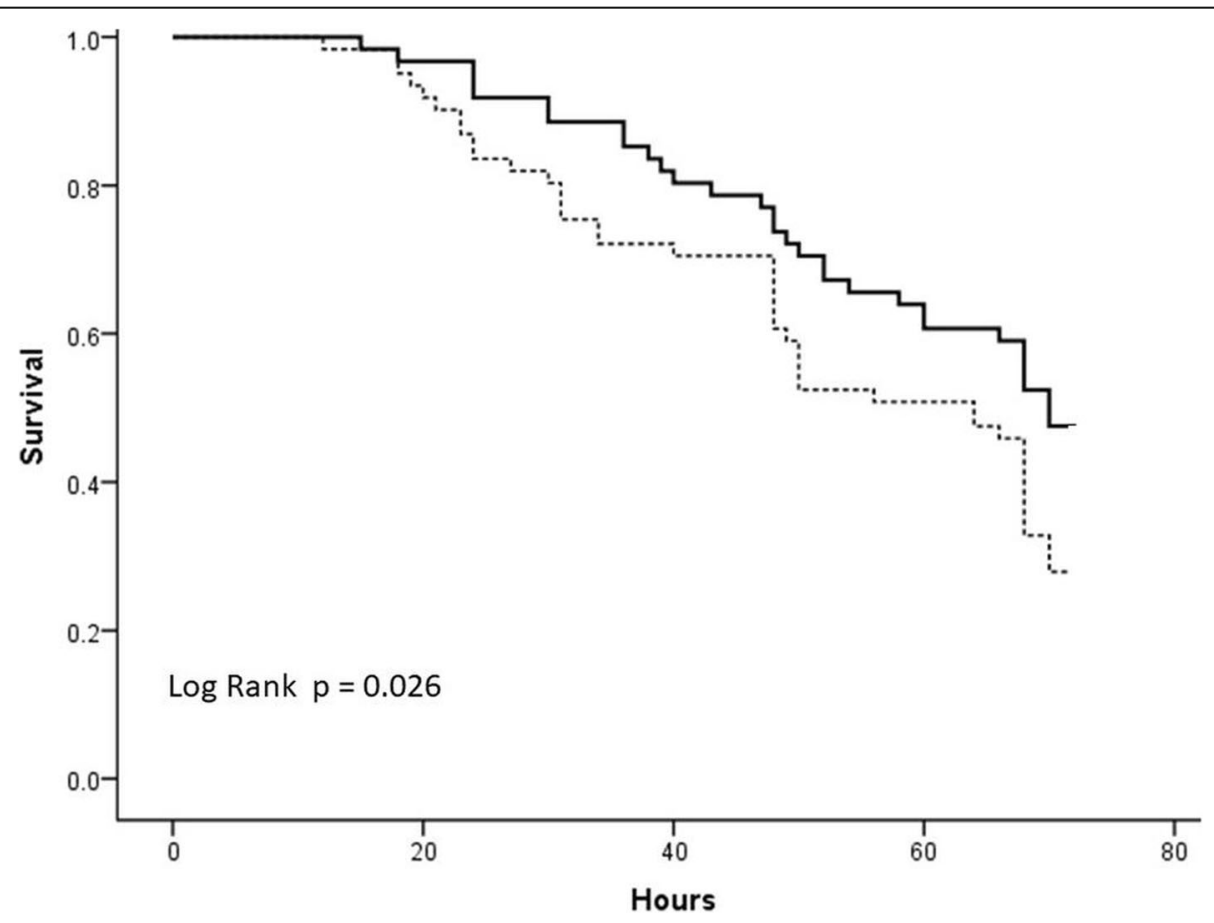

Fig. 1 The Kaplan-Meier circuit survival curve according to diffrent citrate anticoagulation protocol. Survival curves derived from the analysis of $\mathrm{CWH}$ termination for any cause. Continuous line represents fixed protocol, dotted line represents titrated protocol 
Table 3 Reasons for stopping continuous veno-venous hemofiltration

\begin{tabular}{|c|c|c|c|}
\hline & Fixed group & Titrated group & $P$ value \\
\hline \multicolumn{4}{|l|}{ Spontaneous circuit failure, n (\%) } \\
\hline Clotting & $10(15.2)$ & $19(29.7)$ & 0.047 \\
\hline Catheter dysfunction & $3(4.5)$ & $4(6.3)$ & 0.667 \\
\hline \multicolumn{4}{|l|}{ Elective discontinuation, n (\%) } \\
\hline Replaced as planned after $72 \mathrm{~h}$ & $45(68.2)$ & $34(53.1)$ & 0.079 \\
\hline Clinical target achievement & $4(6.1)$ & $3(4.7)$ & 0.729 \\
\hline Death & $3(4.5)$ & $2(3.1)$ & 0.674 \\
\hline Citrate Accumulation & $1(1.5)$ & $2(3.1)$ & 0.541 \\
\hline
\end{tabular}

protocol had less monitoring needs (3.3 [IQR 2.3-4.5] vs. 5.7 [IQR 3.3-6.9], $p=0.001$ ).

\section{Complications}

No new onset bleeding complication attributable to RCA was observed. In terms of metabolic complications, hypernatremia $(5.4 \%, n=7)$ and metabolic alkalosis $(8.5 \%, n=11)$ were common in all patients. The incidence of hypernatremia was comparable between groups (6.1\% in the fixd group vs $4.7 \%$ in the titrated group, $p=$ $0.667)$, while the incidence of metabolic alkalosis was higher in the titrated group ( $14.1 \%$ vs $3.0 \%, p=0.024)$. However, all patients had normalization of serum bicarbonate levels after reducing or discontinuation of alkali infusion. The s-iCa levels $(\mathrm{mmol} / \mathrm{L})$ were relatively stable and there was no difference between groups $(1.02 \pm 0.17$ in the fixed group vs. $1.04 \pm 0.14$ in the titrated group, $p=0.299$ ). Aside from those who presented with hypocalcemia before CVVH, only two patients (one in each group, $p=0.983$ ) presented with asymptomatic hypocalcemia. Moreover, six patients (3 cases in each group, $p=0.969)$ were suspected of CA, and three of them died from refractory shock, the remaining patients were corrected after decreasing the dose of citrate infusion.

\section{Discussion}

The cumbersome program and the shortage of commercial solution hindered the regular application of RCA. In this study, we presented a new protocol using fixed circuit citrate concentration combined with modified algorithm for calcium and buffer supplement, and demonstrated that it improved the circuit lifespan and the convenience while maintaining a similar safety, when compared with the titrated protocol. Using only commercial preparations may contribute to its widespread application.

Our new protocol has different features from the reported constant citrate concentration schemes, which used either a fixed flow rate of blood and citratebuffered solution [13-16] or a constant ratio between them [17-20]. The obvious disadvantages were not only that citrate- buffered solution was not generally commercially available, but also that the adjustment of flow rate was inflexible for the former, and the fixed relationship between citrate and blood flow was uncertain for the latter because RF rate varied with ultrafiltration flow and desired fluid removal $[2,5]$. By contrast, the separate citric infusion overcame these shortcomings, as the anticoagulation effect was not coupled to solute control. Also, the programs published so far have required calcium-free solutions, and when calcium-containing solution as only option, the appropriate citrate concentration was not clear due to an increased citrate load and accumulation risk $[3,5,14]$. Therefore, the present study also explored this issue.

The shorter filter lifespan in the titrated group was likely due to the lower initial citrate concentration (almost $3 \mathrm{mmol} / \mathrm{L}$ of extracorporeal blood), which resulted in a high initial c-iCa level, and the consequent frequent modification of citrate or calcium supplement flow might affect the stability of c-iCa levels (e.g., about $10 \%$ of all measurements wasn't achieved the target range of $0.2-0.4 \mathrm{mmol} / \mathrm{L})[4,5,24]$. On the contrary, target $\mathrm{c}-\mathrm{iCa}$ was easily achieved without further change of citrate flow rate in the fixed group. Therefore, appropriate circuit citrate concentration was prerequisite for maintaining stable target $\mathrm{c}-\mathrm{iCa}$ levels, which was also associated with an adequate circuit lifetime. Moreover, recently published studies suggested that the best effect of anticoagulation occurred when the c-iCa was $<0.35 \mathrm{mmol} / \mathrm{L}$, there was near total inhibition of coagulation and was better than the usual target $(\leq 0.4 \mathrm{mmol} / \mathrm{L})[3,5,25,26]$. In the present study, the median c-iCa level and most of the $\mathrm{c}-\mathrm{iCa}$ levels measured during $\mathrm{CVVH}$ could be maintained at the optimal concentration (i.e., $<0.35 \mathrm{mmol} / \mathrm{L}$ ) in the fixed group, which may partly explain the difference of filter survival between groups. In addition, the circuit duration in our study was longer or similar to those described by others, which reported filter lifespan of 26-70 h using calcium-free RF and citrate concentration of $3-5 \mathrm{mmol} / \mathrm{L}[8-20]$. Because the filter survival was affected by many factors, such as catheter size, 
venous access, blood flow rate and ultrafiltration rates, it was difficult to compare the reasons for the differences between different studies, but it was worth mentioning that the application of LMWH in one-third of our patients (but no difference between groups) may be associated with longer filter lifetime. The latest research by Giani reported that RCA may prove useful also in patients treated with systemic heparinization, as it maximise circuit patency and reduces circuit clotting rate [27]. Of course, the reason for stopping CVVH, such as achievement of clinical target, filter replacement as scheduled or until clotting may also affect the filter lifetime, nevertheless, the rate of circuit clotting in our study was somewhat lower than the reported range of $16-46 \%$ [12-14, 16-18]. Besides, the incidence of suspected CA was $4.6 \%$, which was comparable with the rate of $3-9 \%$ in other studies $[14-17,20]$. These results demonstrated that the circuit citrate concentration of 4 $\mathrm{mmol} / \mathrm{L}$ was effective and safe for predilution calciumcontaining solution.

Our study was the first to compare the convenience of different RCA protocol, and found that the fixed protocol was more convenient in terms of demand reduction for monitoring test and dose adjustment of any supplements. This was mainly due to the fact that the algorithm for calcium and bicarbonate supplements has been improved to replace the traditional on-demand supplementary methods. While the modification of formula was attributed the constant citrate concentrations, which made it possible to use the model to roughly estimate citrate metabolic load, buffers balance and effluent calcium loss $[3-5,20,24]$. Although calcium supplement was very complex, our algorithm was in concordance with some studies, where the calcium infusion rate can be determined according to the extracorporeal calcium loss $[28,29]$. Also, the incidence of metabolic alkalosis was reduced by adjusting the $\mathrm{HCO}^{-}$concentration in the RF. Meanwhile, more effective controls of acid-base status and calcium balance also reduced the need for monitoring test. Particularly, regular measurement of ciCa may be omitted due to almost universally below 0.4 $\mathrm{mmol} / \mathrm{l}$ in the fixed protocol, which may have potential benefits in reducing costs and nurse workload.

We also found that the overall metabolic complications were rather low and easily controlled. On the concerns of $\mathrm{CA}$, in general, shock was claimed to be the risk factor for CA and a contraindication for RCA $[1,12$, 30]. Indeed, clinical practice showed that a considerable proportion of patients with shock could tolerate citrate anticoagulation, especially those if circulation improved [31, 32]. Our results also indicated that the overall incidence of CA even in a significant number of patients with shock or hypoxemia (account for about 90\%) was low.
Several limitations should also be noted. First, due to its historical control group, a longer filter lifespan with fixed protocol may reflect an improvement in the standard of care, but using same CRRT criteria may help to minimize bias. Second, the blood flow rate was usually set between 120 and $150 \mathrm{ml} / \mathrm{min}$ in our institution and further research is needed to evaluate the safety in high blood flow setting, which corresponds to high citrate dose. Third, few patients with severe liver disease were enrolled, who were the most relevant patient group at risk to develop CA, our results must be applied with caution to these patients. Fourth, calcium-containing solution is far from optimal for RCA, however, in case of calcium-free replacement solution shortage, it may be our only option. Lastly, the metabolic load of citrate and calcium homeostasis varied in different CRRT modes, therefore, other modes (e.g., post-dilution CVVH, CVVHD, CVVHDF) needs to be evaluated in future research.

\section{Conclusions}

Our modified fixed citrate concentration protocol seems a feasible, safe, and effective to improve the circuit lifespan and convenience while maintaining a low incidence of metabolic complications when compared to the titrated protocol. Using only commercial preparations that were available in most countries may represent a significant step toward widespread acceptance of RCA.

\section{Abbreviations \\ ACD-A: anticoagulant citrate dextrose solution-A; APACHE: Acute physiology and chronic health evaluation; CA: Citrate accumulation; CRRT: Continuous renal replacement therapy; $\mathrm{CWH}$ : Continuous veno-venous hemofiltration; CWHD: Continuous veno venous hemodialysis; CWHDF: Continuous veno venous hemodiafiltration; c-iCa: Circuit ionized calcium; ICU: Intensive care unit; IQR: Interquartile range; KDIGO: Kidney Disease Improved Global Outcomes; LMWH: Low molecular weight heparin; RCA: Regional citrate anticoagulation; RF: Replacement fluid; s-iCa: Systemic ionized calcium; SHA: Systemic heparin anticoagulation; SOFA: Sequential organ failure assessment}

\section{Supplementary Information}

The online version contains supplementary material available at https://doi. org/10.1186/s12871-021-01319-4.

Additional file 1.

Additional file 2.

Additional file 3 .

Acknowledgements

We thank the staff of the intensive care for their care of patients.

Authors' contributions

All authors contributed to the study conception and design. Material preparation, data collection, and analysis were performed by YS, JMP, HYQ and $\mathrm{BD}$. XYH prepared tables and figures. The first draft of the manuscript was written by YS. All authors read and approved the final manuscript.

Funding

None. 


\section{Availability of data and materials}

The datasets during and/or analysed during the current study available from the corresponding author on reasonable request.

\section{Declarations}

\section{Ethics approval and consent to participate}

This study was approval by the Ethics Committee of the Peking Union Medical College Hospital, Beijing, China (number ZS-950), and written informed consent was obtained from all patients or their relatives.

\section{Consent for publication}

Not applicable.

\section{Competing interests}

The authors declare that they have no competing interests.

Received: 23 November 2020 Accepted: 24 March 2021 Published online: 30 March 2021

\section{References}

1. Khwaja A. KDIGO clinical practice guidelines for acute kidney injury. Nephron Clin Pract. 2012;120(4):c179-84. https://doi.org/10.1159/000339789.

2. Kindgen-Milles D, Brandenburger T, Dimski T. Regional citrate anticoagulation for continuous renal replacement therapy. Curr Opin Crit Care. 2018:24(6):450-4. https://doi.org/10.1097/MCC.0000000000000547.

3. Oudemans-van Straaten HM, Ostermann M. Bench-to-bedside review: citrate for continuous renal replacement therapy, from science to practice. Crit Care. 2012;16(6):249. https://doi.org/10.1186/cc11645.

4. Oudemans-van Straaten HM, Kellum JA, Bellomo R. Clinical review: anticoagulation for continuous renal replacement therapy- heparin or citrate? Crit Care. 2011;15:202.

5. Davenport A, Tolwani A. Citrate anticoagulation for continuous renal replacement therapy (CRRT) in patients with acute kidney injury admitted to the intensive care unit. NDT Plus. 2009:2:439-47.

6. Cui Q, Sun F, Liu S, Xu J, Zhu H, Yu X. A survey of regional citrate anticoagulation for emergency continuous renal replacement therapy. Zhonghua Wei Zhong Bing Ji Jiu Yi Xue. 2020;32(5):595-600. https://doi. org/10.3760/cma.j.cn121430-20200119-00138.

7. Mehta RL, McDonald BR, Aguilar MM, Ward DM. Regional citrate anticoagulation for continuous arteriovenous hemodialysis in critically ill patients. Kidney Int. 1990;38(5):976-81. https://doi.org/10.1038/ki.1990.300.

8. Tobe SW, Aujla P, Walele AA, Oliver MJ, Naimark DM, Perkins NJ, et al. Regional citrate anticoagulation protocol for CRRT using only commercially available solutions. J Crit Care. 2003;18(2):121-9. https://doi.org/10.1053/ jcrc.2003.50006.

9. Gao J, Wang F, Wang Y, Jin D, Tang L, Pan K. A mode of CWH with regional citrate anticoagulation compared to no anticoagulation for acute kidney injury patients at high risk of bleeding. Sci Rep. 2019;9(1):6607. https://doi. org/10.1038/s41598-019-42916-1.

10. Zhang L, Liao Y, Xiang J, Qin W, Wu X, Tang Y, et al. Simplified regional citrate anticoagulation using a calcium-containing replacement solution for continuous venovenous hemofiltration. J Artif Organs. 2013;16(2):185-92. https://doi.org/10.1007/s10047-012-0680-2.

11. Ong SC, Wille KM, Speer R, Tolwani AJ. A continuous veno-venous hemofiltration protocol with anticoagulant citrate dextrose formula a and a calcium-containing replacement fluid. Int J Artif Organs. 2014;37(6):499-502. https://doi.org/10.5301/ijao.5000323.

12. Monchi M, Berghmans D, Ledoux D, Canivet JL, Dubois B, Damas P. Citrate vs. heparin for anticoagulation in continuous veno-venous hemofiltration: a prospective randomized study. Intensive Care Med. 2004;30(2):260-5. https://doi.org/10.1007/s00134-003-2047-X.

13. Nurmohamed SA, Vervloet MG, Girbes AR, Ter Wee PM, Groeneveld AB. Continuous venovenous hemofiltration with or without predilution regional citrate anticoagulation: a prospective study. Blood Purif. 2007;25(4):316-23. https://doi.org/10.1159/000107045.

14. Bihorac A, Ross EA. Continuous venovenous hemofiltration with citrate based replacement fluid: efficacy, safety, and impact on nutrition. Am J Kidney Dis. 2005;46(5):908-18. https://doi.org/10.1053/j.ajkd.2005.08.010.

15. Nurmohamed SA, Jallah BP, Vervloet MG, Yldirim G, ter Wee PM, Groeneveld $A B$. Continuous venovenous haemofiltration with citrate-buffered replacement solution is safe and efficacious in patients with a bleeding tendency: a prospective observational study. BMC Nephrol. 2013;14(1):89. https://doi.org/10.1186/1471-2369-14-89.

16. Leung AK, Shum HP, Chan KC, Chan SC, Lai KY, Yan WW. A retrospective review of the use of regional citrate anticoagulation in continuous venovenous hemofiltration for critically ill patients. Crit Care Res Pract. 2013; 2013:349512.

17. Schilder L, Nurmohamed SA, Bosch FH, Purmer IM, den Boer SS, Kleppe CG, et al. Citrate anticoagulation versus systemic heparinisation in continuous venovenous hemofiltration in critically ill patients with acute kidney injury: a multi-center randomized clinical trial. Crit Care. 2014;18:472.

18. Schmitz M, Taskaya G, Plum J, Hennersdorf M, Sucker C, Grabensee B, et al. Continuous venovenous haemofiltration using a citrate buffered substitution fluid. Anaesth Intensive Care. 2007;35:529-35.

19. Hetzel GR, Schmitz M, Wissing H, Ries W, Schott G, Heering PJ, et al. Regional citrate versus systemic heparin for anticoagulation in critically ill patients on continuous venovenous haemofiltration: a prospective randomized multicentre trial. Nephrol Dial Transplant. 2011;26(1):232-9. https://doi.org/10.1093/ndt/gfa575.

20. Morabito S, Pistolesi V, Tritapepe L, Zeppilli L, Polistena F, Strampelli E, et al. Regional citrate anticoagulation in cardiac surgery patients at high risk of bleeding: a continuous veno-venous hemofiltration protocol with a low concentration citrate solution. Crit Care. 2012;16(3):R111. https://doi.org/1 $0.1186 /$ cc11403.

21. Mariano F, Morselli M, Bergamo D, Hollo Z, Scella S, Maio M, et al. Blood and ultrafiltrate dosage of citrate as a useful and routine tool during continuous venovenous haemodiafiltration in septic shock patients. Nephrol Dial Transplant. 2011;26(12):3882-8. https://doi.org/10.1093/ndt/gfr106.

22. Joannidis M, Oudemans-Van Straaten HM. Clinical review: patency of the circuit in continuous renal replacement therapy. Crit Care. 2007;11(4):218. https://doi.org/10.1186/cc5937.

23. Hetzel GR, Taskaya G, Sucker C, Hennersdorf M, Grabensee B, Schmitz M. Citrate plasma levels in patients under regional anticoagulation in continuous venovenous hemofiltration. Am J Kidney Dis. 2006;48(5):806-11. https://doi.org/10.1053/j.ajkd.2006.07.016.

24. Page M, Rimmelé T, Prothet J, Christin F, Crozon J, Ber CE. Impact of a program designed to improve continuous renal replacement therapy stability. Ann Fr Anesth Reanim. 2014;33(12):626-30. https://doi.org/10.1016/ j.annfar.2014.10.008.

25. Mariano F. Focusing on the basic principles of dialysis to optimize regional citrate anticoagulation. J Crit Care. 2013;28(1):99-100. https://doi.org/10.101 6/j.jcrc.2012.09.001.

26. James MF, Roche AM. Dose-response relationship between plasma ionized calcium concentration and thrombelastography. J Cardiothorac Vasc Anesth. 2004;18(5):581-6. https://doi.org/10.1053/j.jvca.2004.07.016.

27. Giani M, Scaravilli V, Stefanini F, Valsecchi G, Rona R, Grasselli G, et al. Continuous renal replacement therapy in Venovenous extracorporeal membrane oxygenation: a retrospective study on regional citrate anticoagulation. ASAIO J. 2020;66(3):332-8. https://doi.org/10.1097/MAT. 0000000000001003

28. Zheng Y, Xu Z, Fan Q, Zhu Q, Ma S, Lu J, et al. Calcium supplementation in CWH using regional citrate anticoagulation. Hemodial Int. 2019;23:33-41.

29. Kramer L, Bauer E, Joukhadar C, Strobl W, Gendo A, Madl C, et al. Citrate pharmacokinetics and metabolism in cirrhotic and noncirrhotic critically ill patients. Crit Care Med. 2003;31(10):2450-5. https://doi.org/10.1097/01.CCM. 0000084871.76568.E6.

30. Mariano F, Bergamo D, Gangemi EN, Hollo' Z, Stella M, Triolo G. Citrate anticoagulation for continuous renal replacement therapy in critically ill patients: Success and limits Int J Nephrol 2011; 2011:748320.

31. Oudemans-Van Straaten HM, Bosman RJ, Koopmans M, van der Voort PH, Wester JP, van der Spoel II, et al. Citrate anticoagulation for continuous venovenous hemofi Itration. Crit Care Med. 2009;37(2):545-52. https://doi. org/10.1097/CCM.0b013e3181953c5e.

32. Mariano F, Tedeschi L, Morselli M, Stella M, Triolo G. Normal citratemia and metabolic tolerance of citrate anticoagulation for hemodiafiltration in severe septic shock burn patients. Intensive Care Med. 2010;36(10):1735-43. https://doi.org/10.1007/s00134-010-1909-2.

\section{Publisher's Note}

Springer Nature remains neutral with regard to jurisdictional claims in published maps and institutional affiliations. 